\title{
Investigations of Friction Stir Welds between 5083 and 7075 Aluminum Alloys Using EBSD and X-Ray Techniques
}

\author{
I. KALEMBA-ReC*, M. WróBel And M. KopyŚCIAŃSKi \\ AGH University of Science and Technology, Faculty of Metals Engineering and Industrial Computer Science, \\ al. A. Mickiewicza 30, 30-059 Krakow, Poland

\begin{abstract}
Microstructure and texture of the friction stir welded 5083 and 7075 aluminum alloys were characterized by means of the electron backscatter diffraction and X-ray techniques. A complex vortex-like microstructure with distinctive bands of the both joint materials was found in the centre of the weld. The alternating bands filled roughly equiaxed dynamically recrystallized grain volumes. The grain size was slightly smaller in bands of the alloy 7075 than in bands of the alloy 5083. Grains in the thermomechanically affected zone were elongated, especially on the retreating side of the weld (7075 alloy). On the other hand, the grain shape in the heat affected zone was similar to that for the base material.
\end{abstract}

DOI: 10.12693/APhysPolA.130.996

PACS/topics: $81.20 . \mathrm{Vj}, 81.40 .-\mathrm{z}, 81.40 . \mathrm{Ef}$

\section{Introduction}

The aerospace, automotive, and shipbuilding industries require reliable joints between different metallic materials. Several methods may be applied for dissimilar joining. However, properties of adhesive bonds are often not sufficient, riveting is expensive and traditional welding significantly changes the functional properties because of the formation of hard and brittle intermetallic phases in the weld and its vicinity. The friction stir welding (FSW) process can eliminate or minimize these disadvantages. FSW is a solid-state joining technology. During this process the material is mixed and undergoes intensive plastic deformation accompanied by a limited increase in the temperature. Deformation and thermal phenomena lead to the characteristic changes in microstructure and properties that are much more tolerable than those produced by traditional welding $[1,2]$.

The studies on friction stir welded dissimilar aluminum 5083 and 7075 alloys are not numerous and have been focused mainly on mechanical properties $[3,4]$ or microstructural characteristic of weld cross-sections [5]. This research addresses the characterization of microstructure (grain size, grain boundary characteristics and phase identification) as well as texture development in friction stir welded 5083 and $7075 \mathrm{Al}$ alloys. The investigation was carried out on a material layer parallel to top surface of joined plates. The electron backscatter diffraction (EBSD), energy-dispersive X-ray spectroscopy (EDS) and X-ray diffraction (XRD) techniques were used for the characterization. The typical regions of FSW joints, i.e., the stirred zone (SZ), the thermomechanically affected zone (TMAZ) and the heat affected zone (HAZ) were characterized on the advancing and retreating sides.

*corresponding author; e-mail: kalemba@agh.edu.pl

\section{Experimental}

$6 \mathrm{~mm}$ thick plates of $\mathrm{Al}-\mathrm{Mg}$ alloy 5083-H111 and $\mathrm{Al}-$ $\mathrm{Zn}-\mathrm{Mg}-\mathrm{Cu}$ alloy $7075-\mathrm{T} 651$ were friction stir welded at the Welding Institute in Gliwice (Poland). The chemical composition of joined alloys is shown in Table I. The welding process was carried out using a tool consisting of a shoulder made of a high speed steel and the Triflute pin. The selected process parameters (tool rotation speed of 355 rotations per min and welding velocity of $140 \mathrm{~mm} / \mathrm{min}$ ) as well as the weld configuration (the 5083 alloy on the advancing and the 7075 alloy on the retreating side) allowed for production of defect-free joints. The weld line direction was parallel to the extrusion direction of the joined plates. The top view of the produced weldment and its cross-section is shown in Fig. 1a.

TABLE I

Nominal chemical composition [wt\%] of investigated alloys

\begin{tabular}{c|c|c|c|c|c|c|c|c|c}
\hline \hline alloy & $\mathrm{Mg}$ & $\mathrm{Zn}$ & $\mathrm{Cu}$ & $\mathrm{Mn}$ & $\mathrm{Si}$ & $\mathrm{Fe}$ & $\mathrm{Cr}$ & $\mathrm{Ti}$ & $\mathrm{Al}$ \\
\hline 5083 & 4.32 & 0.04 & 0.05 & 0.56 & 0.16 & 0.36 & 0.06 & 0.05 & bal. \\
7075 & 2.56 & 6.18 & 1.86 & 0.02 & 0.13 & 0.15 & 0.21 & 0.04 & bal.
\end{tabular}

The top surface of the weld (in contact with the tool shoulder) was examined by means of a combined EBSD/EDS analysis and XRD techniques. The XRD allowed for identification of constituent phases and the crystallographic texture measurement. Samples for the investigation were prepared in a conventional way. Initially, the external layer of material with significant roughness was removed by grinding on sandpapers until smooth surface was obtained. Then samples were mechanically polished with a colloidal silica solution. Samples for the XRD examination were etched by the Keller reagent. The weld top surface prepared for examination is showed in Fig. 1b together with marked points of measurements. A Nova NanoSEM 450 FEG microscope 
equipped with a combined EBSD/EDS system operating at $15 \mathrm{kV}$ was used to characterization of microstructure. An EBSD/EDS maps were collected with a step $1 \mu \mathrm{m}$. The limit of the precision of EDS analysis (including all sources of errors) is $\pm 2 \%$. The TSL-OIM ${ }^{T M}$ analysis software was used for the analysis. The XRD measurements were performed on the PANalytical-Empyrean diffractometer. The $\mathrm{Cu} K_{\alpha}$ radiation was used and the exposed region had dimensions ca. $2 \times 1.6 \mathrm{~mm}^{2}$.

Additionally the Vickers hardness measurements were carried out on polished top surface of weld at the load of $9.81 \mathrm{~N}$ and at indentation time of $10 \mathrm{~s}$.

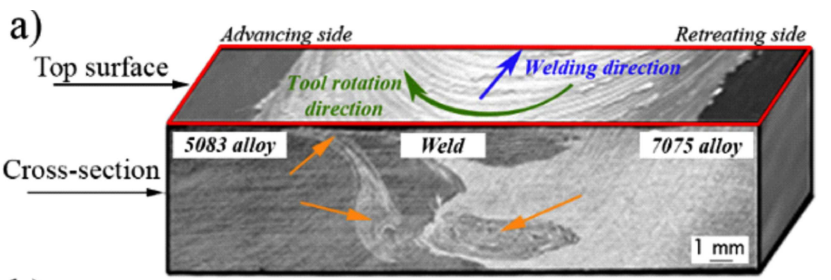

b)

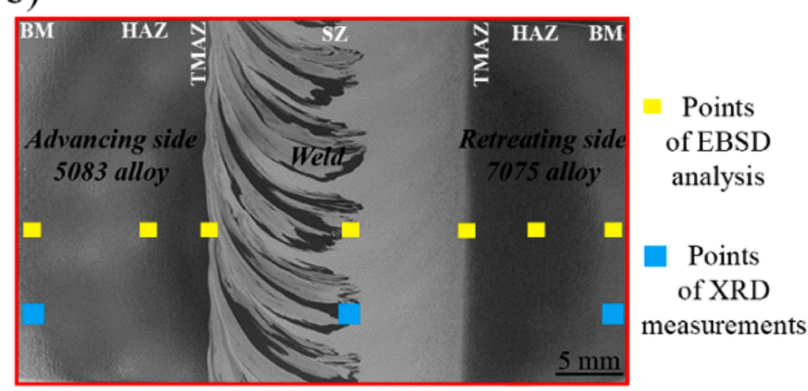

Fig. 1. The examined weld: overall view (a part of the SZ fragmented into bands are marked by arrows) (a); top weld surface with marked areas of analysis (b).

\section{Results}

The orientation imaging microscopy (OIM) maps of the TMAZ and HAZ are shown in Fig. 2. Additionally the percentage of low-angle boundaries (LABs) with misorientation of $2-15^{\circ}$ and high-angle boundaries (HABs) with misorientation angles greater than $15^{\circ}$ are shown in the insets of each figure. The joining alloys differ in grain shape and size. The alloy 5083 exhibits equiaxed and relatively small grains (size about $50 \mu \mathrm{m}$ ). On the other side, in the alloy 7075 , the grains were elongated along the extrusion direction and its size was about four times larger. The microstructure of the heat affected zone and the base material was similar. The ratio of LABs and HABs in HAZ on the advancing side was the same as for the base material. In contrast, on the retreating side the fraction of HABs decreased significantly, i.e. it was lower by about $20 \%$. The TMAZ is a region where the microstructure is modified by the plastic deformation and heat effects associated with the FSW. Significantly elongated grains (aspect ratio about 5) and the LABs frequency above $50 \%$ were typical for this region.

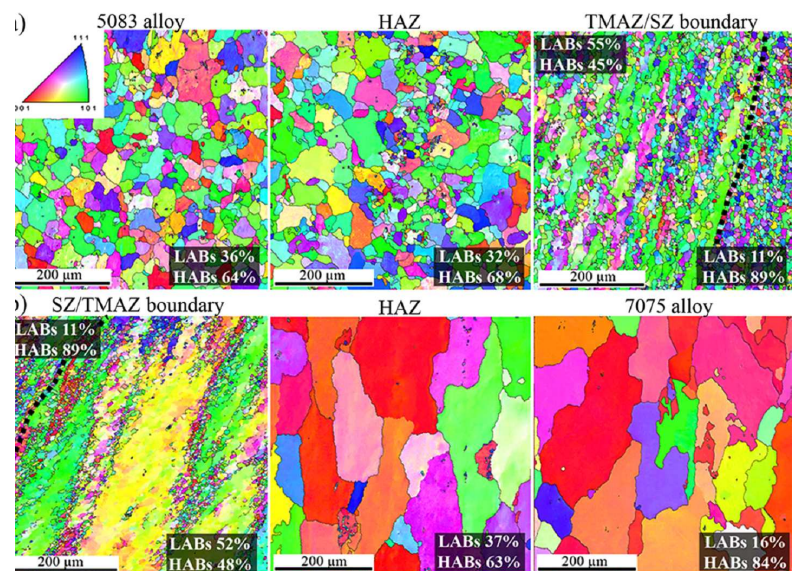

Fig. 2. Orientation imaging maps of the base materials, HAZs, TMAZ and SZ on the advancing side (a), on the retreating side (b); insets in figures show the ratio of low-angle boundaries and high-angle boundaries.
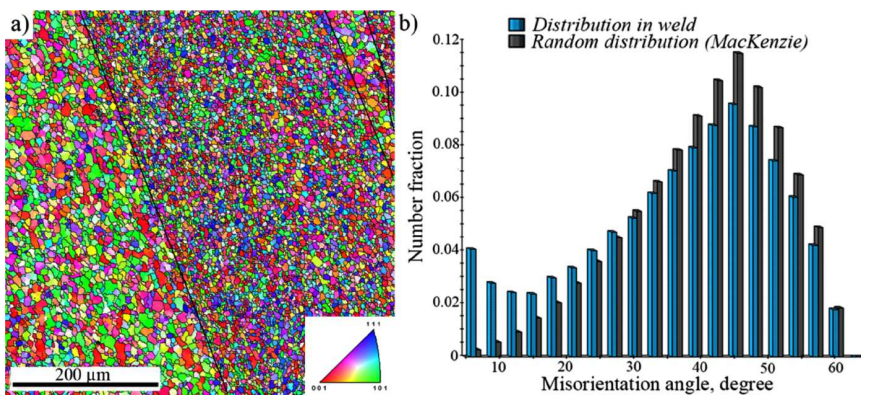

Fig. 3. Orientation imaging map of the stirred zone (a), boundary misorientation distributions (b).

A part of the SZ, marked by arrows in Fig. 1a, was fragmented into alternating bands of different grain size (Fig. 3). The bands were formed in the SZ region where the harder 7075 alloy was pushed by tool into the softer 5083 alloy. The hardness profile is shown in Fig. 4. Bands of a small grain (BSG) size material (about $4 \mu \mathrm{m}$ ) were separated by bands with a slightly larger grain (BLG) size, about $6 \mu \mathrm{m}$. However, the grains in the both type bands were roughly equiaxed. It is worth mentioning that the band-like microstructure is typical for plastically deformed metals [6-8]. EDS maps showing the chemical

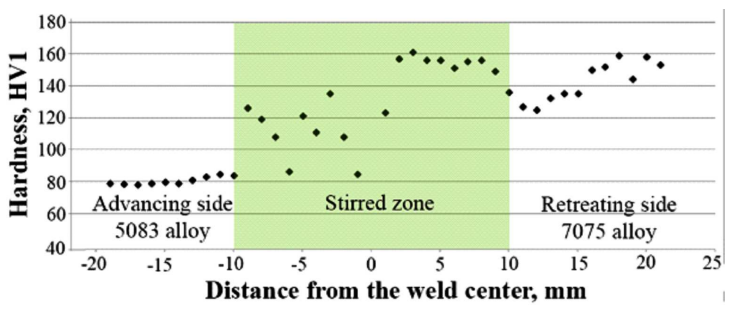

Fig. 4. Hardness profile, top surface of weld. 

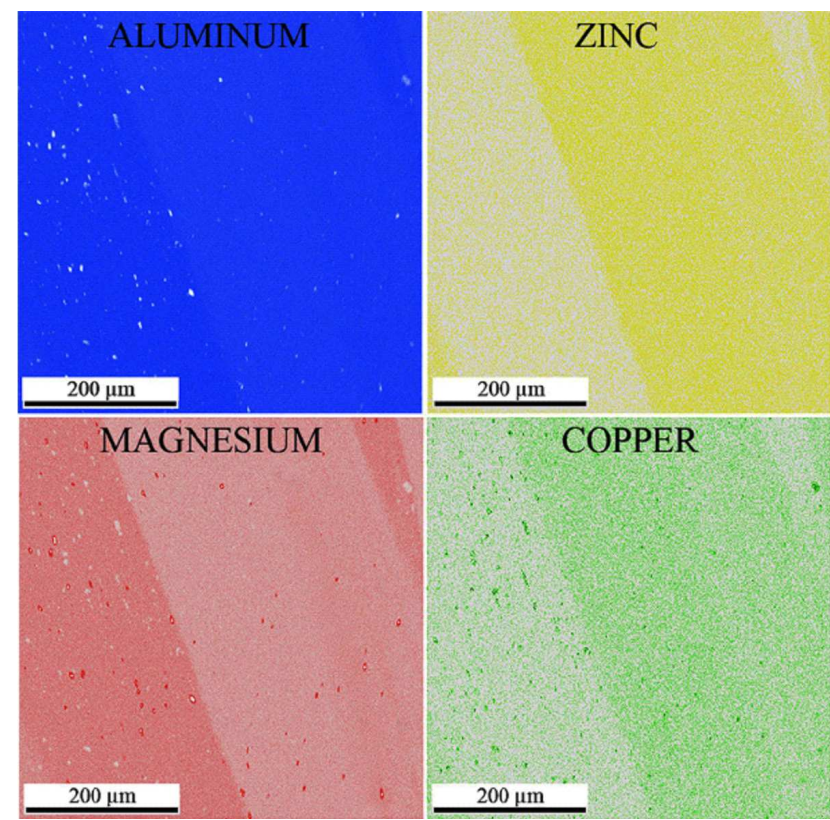

Fig. 5. Elements distribution (EDS) in the stirred zone shown in Fig. 3.
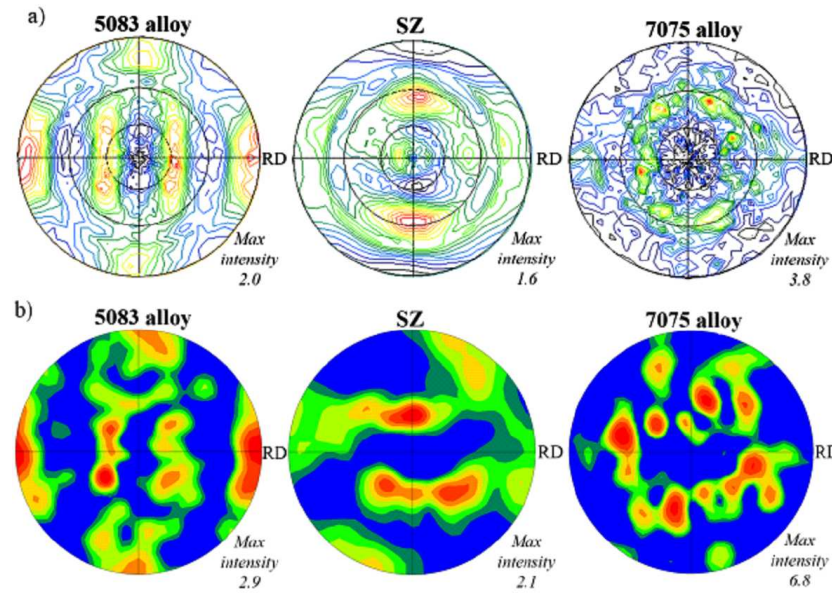

Fig. 6. $\langle 111\rangle$ pole figures for base materials and their weld recorded by XRD a), EBSD (b).

elements distribution are presented in Fig. 5. It is apparent that BSGs are enriched in $\mathrm{Zn}$ and $\mathrm{Cu}$ and simultaneously depleted in $\mathrm{Mg}$. The element distribution in the BLGs was opposite. This proves that the BSG and BLG were formed by the 7075 and 5083 alloys, respectively. It should be mentioned that the grain size for the base material of the alloy 7075 was significantly larger than for the alloy 5083 (see Fig. 2). It is believed that the stronger grain refinement in the alloy 7075 resulted from precipitation processes accompanying the dynamic recrystallization occurring during the FSW process.

The distribution of grain boundaries misorientation in the SZ slightly deviates from the Mackenzie distribution
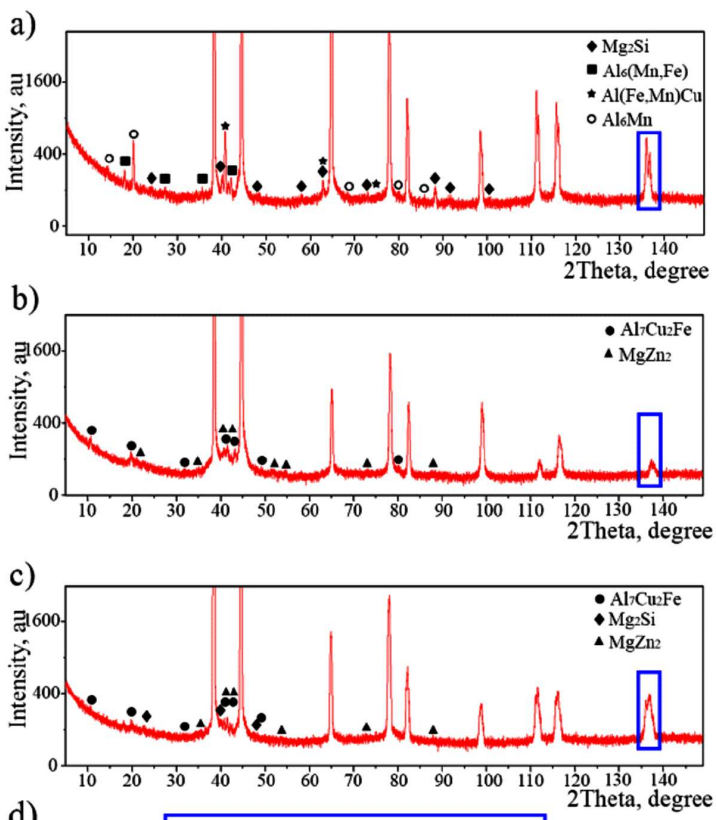

d)

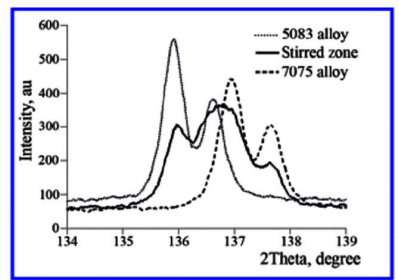

Fig. 7. The XRD patterns of 5083 alloy (a), 7075 alloy (b), stirred zone (c), comparison of all spectra in the range marked a blue rectangle $(\mathrm{d})$.

function that is typical for randomly textured polycrystalline metals with the cubic point symmetry [9]. It indicates on a relatively weak crystallographic texture of the SZ as was confirmed by the X-ray measurements (Fig. 6a). Figure 6a also shows the difference between the SZ texture and the textures of both base materials. Such a transformation of the texture likely results from the recrystallization. Additionally, the dynamic recrystallization that occurs in the SZ leads to reduction in the grain size.

The similarity of the X-ray pole figures and pole figures calculated from the EBSD data (Fig. 6b) indicates that the examined regions are large enough to be representative for entire volume.

The XRD patterns are shown in Fig. 7. The nonlabeled peaks shown in this figure belong to $\mathrm{Al}$. The XRD patterns revealed several phases in the investigated materials. Therefore, the alloy 5083 contains $\mathrm{Mg}_{2} \mathrm{Si}$, $\mathrm{Al}_{6}(\mathrm{Mn}, \mathrm{Fe}), \mathrm{Al}(\mathrm{Fe}, \mathrm{Mn}) \mathrm{Cu}$ and $\mathrm{Al}_{6} \mathrm{Mn}$ phases (Fig. 7a). On the other hand, the $\mathrm{Al}_{7} \mathrm{Cu}_{2} \mathrm{Fe}$ and $\mathrm{MgZn}_{2}$ phases were found in the alloy 7075 (Fig. 7b). Some phases that were present in both base alloys, i.e., $\mathrm{Al}, \mathrm{Al}_{7} \mathrm{Cu}_{2} \mathrm{Fe}, \mathrm{Mg}_{2} \mathrm{Si}$ and $\mathrm{MgZn}_{2}$ were found also in the SZ (Fig. 7c). The selected range in the XRD patterns (Fig. $7 \mathrm{a}-\mathrm{c}$ ) is shown at higher magnification in Fig. 7d. A relative shift in the 
position of $\mathrm{Al}$ peaks from both base materials indicates differences in the lattice parameter of both alloys. The $\mathrm{Al}$ peaks of both alloys can be distinguished in the SZ. This is fully consistent with results of the microstructure investigation.

\section{Summary}

The present research showed that the stirred zone of the friction stir welded joints between the 5083 and 7075 alloys consists of the alternating bands formed by both alloys. The bands are characterized by the different grain size. These bands result from mechanical mixing of joining metals. Their microstructure was formed during the dynamic recrystallization process. Significantly elongated grains were observed in the thermomechanically affected zones. However, only slight changes in microstructure of the base materials were observed in the heat affected zone.

\section{Acknowledgments}

This work was supported by the Polish National Science Centre, project No. DEC-2012/07/D/ST8/02737.

\section{References}

[1] F. Micari, G. Buffa, S. Pellegrino, L. Fratini, Procedia Eng. 81, 74 (2014).

[2] N. Kumar, W. Yuan, R.S. Mishra, Friction Stir Welding of Dissimilar Alloys and Materials, Elsevier, Oxford 2015 .

[3] M.H. Shojaeefard, R.A. Behnagh, M. Akbari, M.K.B. Givi, F. Farhani, Mater. Des. 44, 190 (2013).

[4] M. Saeidi, B. Manafi, M.K. Besharati Givi, G. Faraji, Proc. Inst. Mech. Eng. B J. Eng. Manufact. 1, 1 (2015).

[5] I. Kalemba, M. Kopyścianski, Solid State Phenom. 231, 93 (2015).

[6] M. Blicharski, S. Dymek, M. Wróbel, J. Mater. Process. Technol. 53, 75 (1995).

[7] S. Dymek, M. Wróbel, M. Dollar, M. Blicharski, J. Microsc. (Oxf.) 224, 24 (2006).

[8] M. Wróbel, S. Dymek, M. Blicharski, Scr. Metall. Mater. 35, 417 (1996).

[9] J.K. Mackenzie, Biometrica 45, 229 (1958). 